This Technical Report is also published as Chapter 41 IN

M-Health: Emerging Mobile Health Systems, Robert H. Istepanian, Swamy Laxminarayan, Constantinos S. Pattichis, Editors, 2006.

Springer. $624 \mathrm{p}$.

ISBN: 0-387-26558-9

AS

TELEMATIC REQUIREMENTS FOR EMERGENCY AND DISASTER RESPONSE DERIVEDFROM ENTERPRISE MODELS

I. Widya, P. Vierhout, V. M. Jones, R. Bults, A. van Halteren, J. Peuscher, and D. Konstantas 


\section{TELEMATIC REQUIREMENTS FOR EMERGENCY AND DISASTER RESPONSE DERIVEDFROM ENTERPRISE MODELS}

I. Widya, P. Vierhout, V. M. Jones, R. Bults, A. van Halteren, J. Peuscher, and D. Konstantas ${ }^{*}$

\section{INTRODUCTION}

One of the prime objectives of disaster response management is to gain control of the disaster situation as rapidly as possible. Observations have shown that coordination and communication between response teams during disasters is essential for gaining control of the situation, but is often inadequate in past disasters (Auf der Heide, 1989) and often suffers from up-scaling difficulties. Examples were the September $11^{\text {th }}$ attack in New York in 2001 (BBC News, 2002) and the explosion on the premises of a professional firework assembly site, which completely destroyed or severely damaged some 480 houses and some 100 small-medium sized enterprises in the town of Enschede in the Netherlands in 2000 (Oosting, 2001).

The different teams involved in disaster response not only have their own specialties, responsibility and (hierarchical) scope of authority, they frequently differ in their ways of working. The Enschede disaster response, for example, involved several fire brigades (also from neighboring areas), site controlling police teams, crisis coordination teams and medical emergency services teams, which moreover received assistance from across the border in Germany. This scenario illustrates the

\footnotetext{
I. Widya, V.M. Jones, R. Bults, A. van Halteren, and D. Konstantas, Centre for Telematics and Information Technology, Faculty of Electrical Engineering, Mathematics and Computer Science, University of Twente, the Netherlands. P. Vierhout, Faculty of Business, Public Administration and Technology, Univ. of Twente and J. Peuscher, Twente Medical Systems International, the Netherlands.
} 
fact that a disaster response process has to be viewed as a complex and multifaceted distributed system involving many teams and requiring sophisticated coordination and communication facilities.

Disaster response needs to be dynamic and adaptive. It has to cope with a range of events, from near disasters to very large-scale actual disasters involving a chain of rapidly escalating accidents. It also has to cope with dynamic (rescue) processes. Previous disasters have shown, on one hand, that many off-duty rescue services staff spontaneously volunteers at the site of the accident. The inability to coordinate the many off-duty volunteers was explicitly identified as a problem in a report on the New York September $11^{\text {th }}$ disaster. To make best use of these extra resources, the volunteers need to be identified, coordinated and deployed effectively within team structures. On the other hand, communication infrastructure may be severely damaged or congested at the scene of the disaster. However, due to the advancement of Information and Communication Technology (ICT) several communication alternatives may be available, e.g. GSM, UMTS, terrestrial and satellite links, and including ad-hoc or rapidly deployable networks (Midkiffs and Bostian, 2002). These (rapidly deployable) communication infrastructures and alternatives are valuable in disaster response (Doarn et al., 2004).

A major challenge in medical emergency services and disaster response is how to improve the management process using ICT. For example, how to select dynamically the computing and network resource alternatives (which possibly are scarcely available at a scene of a disaster) that match with the coordination and communication needs of the medical emergency or disaster response teams.

This chapter explores the potential of mobile telematic services to support medical emergency and disaster response, in particular it addresses the requirements of the communication support for medical emergency or disaster response teams.

Requirements of telematic services for disaster response have been investigated for example in Meissner (2002). In this chapter, we derive the requirements using Enterprise Models. We apply a primarily top-down approach using the notion of viewpoints introduced in the ODP (Open Distributed Processing) framework (Blair and Stefani, 1998). We start with Enterprise models for their emphasis on justification purposes. Particularly, we describe the models using generic organizational notions like tasks, roles and teams which are responsible for the tasks, and agents who act in certain roles to perform the tasks. These Enterprise models are inspired by the educational models describe in Widya (2002) and the model for healthcare processes described in Luzi (1997). This healthcare model however defines a guideline-oriented conceptual model of healthcare units for workflow formulations of healthcare processes and healthcare process reengineering. Instead of addressing the details of tasks and inter task relations relevant for workflow and process reengineering, we focus on the (refined) modeling of teams and agents. This 
focus meets our objective to derive the requirements of telematic services that match the communication needs in disaster response. However, readers interested in disaster management information systems and tools may consult FEMA (2001).

Furthermore, the Enterprise models have been specified in a way to capture the invariants of healthcare and disaster response processes and for this reason it can be viewed as a generic meta-model in the context of object-oriented technology (Holz et al., 2001). We therefore believe that our model can be specialized for different disaster specialties. We validate our Enterprise models by applying them to derive the requirements for emergency services of the tele trauma team trial of the European project MobiHealth (Jones et al., 2004).

The Enterprise models proposed also separate roles responsible for the tasks from the agents assigned to those roles. This separation of functional entities from the physical entities has the advantage that roles can be virtually (and immediately) moved to the scene of the disaster without the need to transport all agents to the scene. This is a simple form of an augmented reality environment (Azuma, 1997; Milgram and Kishino, 1994). It brings on-site reality at the scene of the disaster into the scope of command and control of off-site teams or team members. In emergency services, trauma specialists (i.e. agents) at care centers may retain their established way of working as much as possible if augmented reality brings the scene of accidents to their scope of monitoring and control. This property is essential for the acceptance of ICT deployment as added value services, for example if compared to the deployment of significant different ways of working.

The remainder of the chapter is organized as follows. The next section discusses the Enterprise models. For validation of the models several issues of healthcare and disaster scenarios are presented in Section 3. Section 4 discusses the requirements for the telematic support which can be derived from the models and which conforms to the scenarios. Finally, Section 5 provides the conclusion of this chapter.

\section{ENTERPRISE MODEL}

This section describes the Enterprise models for disaster response and healthcare processes (in particular, emergency services). We use the Unified Modeling Language (UML) (Booch et al., 2001) to express these models. First, we analyze the considered processes from an organizational enterprise perspective to achieve a generic model which can cope with a broad range of emergency or disaster events. Then, we refine the model further to reveal event specific modeling details like the dependencies between modeled entities which instantiations are distributed in the augmented reality environment, for example Body Area Networks (BANs) of patients' vital sign sensors in emergency events (Jones et al., 2004) or BANs of firefighters in disaster response (Meissner et al., 2002). In the context of the ODP 
framework, this means that the refined models possess properties of a Computational viewpoint model. Further elaboration of these dependencies will in turn reveal the requirements of the communication support for emergency services or disaster response (cf. requirements for ODP Engineering viewpoint model).

\subsection{Task Structure}

In this chapter, a healthcare process is viewed as a workflow of objective-driven tasks aimed to improve or to restore a patient's condition and, conversely, a task is an embodiment of healthcare activities (Luzi et al., 1997). Similarly, disaster response is viewed as a workflow of objective-driven tasks aimed to control disasters.

In a typical healthcare scenario, we can distinguish between diagnostic tasks and treatment tasks (Hobsley, 1979). Moreover, different diagnostic approaches can be applied in healthcare. The selected approach strongly influences the diagnostic task and the breakdown of this task. In the differential diagnosis approach, a diagnosis is labelled in terms of the pathological disease. The list of diseases matching the symptoms and signs in a previous diagnostic stage has to be narrowed-down, for example using additional tests. This may eventually lead to the cause. On the other hand, in the working diagnostic approach, a diagnosis is labelled in terms of symptoms and signs. Refinement is then sought in order to determine the subsequent activities. A sequence or a partial order of diagnostic activities refines the diagnosis towards the etiological or pathological cause. Preference for a certain approach depends amongst others on the medical specialty.

If the diagnostic hypothesis is confirmed, a treatment task may be initiated. This task may also be decomposed into several subtasks, e.g. treatment planning, execution and evaluation. These (sub-) tasks are often chronologically intertwined with the diagnostic tasks, since the validity of a diagnosis may need to be monitored continuously as this validity affects the treatment and moreover patient illness may develop in other directions.

Similar structure of tasks can be identified for disaster management. Disaster response has to be prepared in a planning phase (disaster preparedness in FEMA (2001)). Intertwined tasks across different disaster management phases have been identified by Auf der Heide (1989), since disasters are different and traditional division of activities and resources of routine emergency management are often unsuitable.

\section{Hierarchical Task Structure:}

An Enterprise model which can cope with hierarchically structured tasks is needed for the modelling of diagnostic processes which apply the differential approach and also for the modeling of inter- and intra-organizational disaster 
response processes. Moreover, if the model also can cope with partially ordered tasks, it will also be suitable for diagnostic working approaches. The need to cope with both kinds of structuring of tasks has been identified in other domains, e.g. in task-oriented educational processes (Widya et al., 2002) and enterprise models of telecommunication network architectures (Yates at al., 1997). Accordingly, we reuse those task structures to model healthcare tasks as well as scalable disaster response tasks (Figure 1). For clarity of the figures, methods and attributes of classes are not shown in the UML class diagrams.

Figure 1 shows that a task is either a leaf-task or a true compositional aggregation of tasks. The aggregated tasks are constrained by the association class compositional-constraint, which for example represents the constraints on the permitted partial order of these tasks, on the diagnostic narrowing of the differential approach or on the decomposition of the medical objective of the encapsulating task.

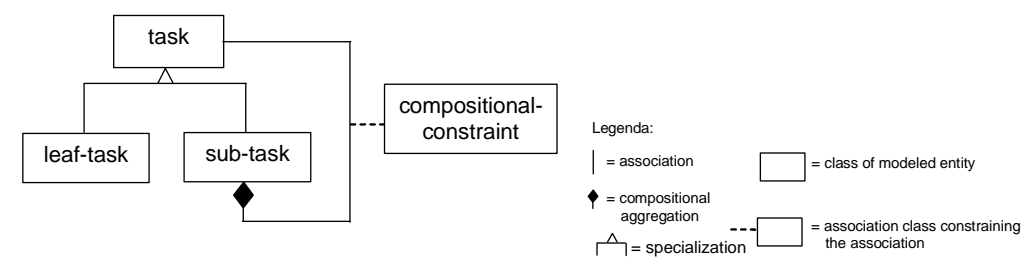

Figure 1 Healthcare and disaster response task structure

The model can cope with arbitrarily deeply nested tasks, including flatly structured tasks. The hierarchical depth of a task structure not only depends on the complexity of the task modelled, it also depends on the task context such as the urgency of the task. The following citation illustrates the need for a flat, monolithically structured task, which contains a large set of activities in a complex medical emergency scenario (Argyle, 1996):

\begin{abstract}
"In dealing with the trauma victim, the physician must treat as he or she gathers information. The approach cannot be routine "take a history, do an exam, order some tests, make a diagnosis, then treat the patient." Therapeutic interventions must be made "on the fly," before the full evaluation can be completed. ......... For example, the combination of low blood pressure, unilaterally decreased breath sounds, and respiratory distress triggers a response from the physician. A chest tube is placed immediately, rather than waiting until an x-ray can "prove" the diagnosis."
\end{abstract}

The flexibility of the model in respect of the hierarchy and the intertwining of tasks makes the model suitable for disaster management tasks. These tasks have to cope dynamically with a range of scenarios, scalable from near accidents to large disasters involving chains of accidents, for which urgency may also be essential. 


\section{Refined Healthcare Task Structure:}

In the context of healthcare, a leaf-task embodies the work-item activities associated with the care of a patient's health, for example observation, interview, preparation, treatment or hypothesis validation activities. An activity of a task may require resources such as oxygen saturation, ECGs and blood pressure data (modelled by class resource and its components in the refined model of tasks shown in Figure 2 and Figure 4, respectively). It may also require (logistical) facilities such as an operating theatre or an intensive care unit (class facility). From the perspective of a medical specialty, a patient is a subject which receives care of the specialty at a reference point (i.e. a particular time and location). A component of leaf-task is therefore care-receiver, which has to be assigned to a patient by the association class patients'-schedule, which contains the reference point.

The model also reveals the modelling strength of UML compositional aggregations, for instance if the treatment task is an operation, the instantiation of the leaf task components guide to plan a facility (i.e. an operation theatre). On the other hand, if the association class patients' schedule is not properly implemented by corresponding data flow diagrams or medical protocols, patients scheduling will be sensitive to human assignment mistakes like authentication mismatch between a task and a scheduled patient. Therefore, the class patients' schedule needs to be dependent (in UML sense) on scheduling policies which refer to medical protocols (e.g. provide patients with identification labels, check the labels before treatment and check patients' name) or authentication protocols (this dependence is not shown in the figure).

In a firefighting scenario, resources of on-site firefighters' tasks may represent resident register information, building or site maps and facility may represent tools such as voice conference or e-messaging applications for command and control guidance from an off-site command and control center (cf. Meissner et al., 2002). Disaster response leaf-tasks do not posses a care-receiver component, but instead a deliverable component (not shown in the figure) which represents results of a task in an information system sense. For example, firefighting reports which may further be used as resources of consecutive tasks (i.e. assigned via an association class to a consecutive leaf-task). On the other hand, resources of off-site leaf-tasks in a firefighting scenario may represent live video clips of the site of the fire, processed read-outs of on-site sensors measuring gas compound emissions or casualty statistics. Without being present at the scene of the accident, the off-site assisting or controlling team members, which depends on remotely located resources, may therefore extend their scope of assistance and control onto the scene of the accident in the augmented reality environment. 


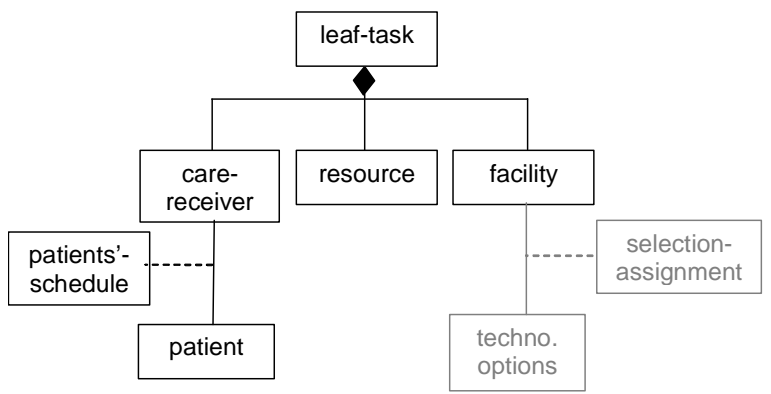

Figure 2 Leaf task structure and patient-task assignment

\subsection{Task - Team Structure}

As with educational tasks (Widya et al., 2002), disaster management or healthcare tasks are organized and performed by agents acting in certain roles and in these roles these agents are responsible for the tasks. In healthcare, the class role represents a medical role (e.g. an expert in anaesthesia) and eventually has to be assigned to an agent on duty (e.g. an anaesthetist on duty). In the assigned role, this agent has the responsibility to perform a certain medical task with the authorization rights associated to the role (represented by authorization in Figure 3). In disaster response, these assignments and authorization may need to be specified or updated dynamically during disaster response, due to the different conditions of disasters and pre-planned activities of tasks in disaster preparedness process may be unsuitable (Auf der Heide, 1989). One may view this issue as an overlapping of phases, the disaster preparedness and the disaster response.

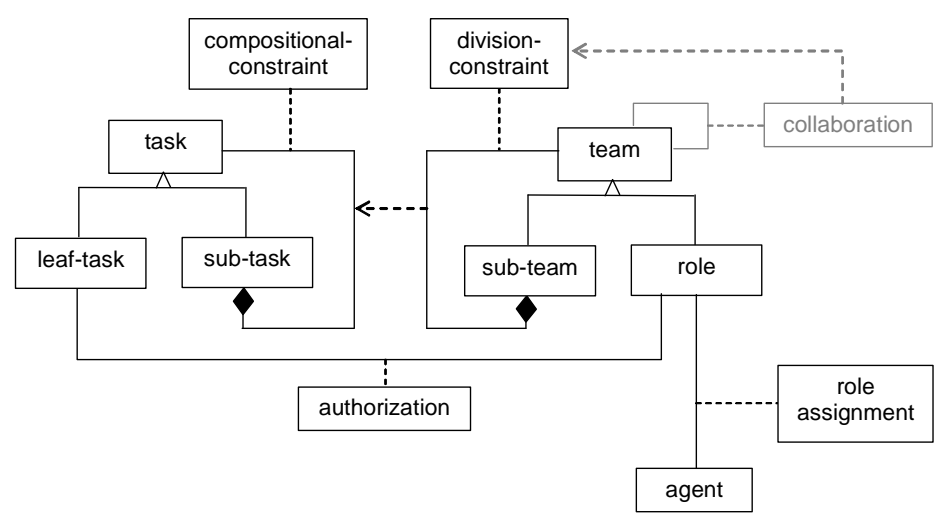

Figure 3 Task - Team structure for healthcare processes and disaster response 
Since complementary roles can split responsibility for a task, especially for complex and aggregated tasks, we introduce the class team either as a role or a compositionally aggregated team. The aggregation is constrained in a multi-way manner by the association class division-constraint. In healthcare for example, division-constraint may impose how a medical assistant role relates to the role being assisted and specify e.g. whether an assistant anaesthetist at a point of care has the right to place a chest tube in a trauma case when the off-site anaesthetist is at the hospital. In disaster management, division-constraint may impose the use of a common frequency band and radio technology to enable communication between teams of different disciplines (e.g. fire brigade, police, medical teams). Add-on guidelines or policies for coordination and communication may also be incorporated in this association class, for example the preferred set of communication tools and technology.

In large-scale accidents furthermore, several teams may perform the same task regime concurrently, for example in disasters where a number of rescue teams are deployed and where each team follow the same rescue regime and policies (the black dashed arrow in Figure 3 models this property).

\subsection{Team-Team Structure}

The association class collaboration (piggybacked in grey in Figure 3), which is a refined part of task-team structures, specifies how teams are related to one another. Amongst others it represents the coordination and communication aspects between teams as is imposed by the association class division-constraint (dashed arrow in the figure). For example, the provisioning of a BAN incorporating communication devices tuned on a specific frequency band or wireless technology as imposed or prioritized by division-constraint. The grey-colored UML construct also models the breakdown of the (multiparty) association class division-constraint into the simpler association class collaboration.

Due to the nested structure of teams, collaboration also represents the intra-team coordination and communication, e.g. referring the rescue protocols that should be used between an on-site agent at a point of care and an off-site agent at the control center, e.g. as illustrated earlier by the healthcare case involving the on-site assistant anaesthetist and the off-site anaesthetist. The class facility of a leaf-task (Figure 2) represents the agreed communication tool, including its configuration setting.

\subsection{Task Resource and BANs}

The resources of a task represent for example ECGs in emergency services or chemical emission graphs in firefighting. These resources typically depend on the context of the task, for example, the objective of the task (e.g. validate cardiac arrest 
or validate rescuer safety) and indirectly the role responsible for the task. Since roles are conceptual modelling entities and the agents assigned to these roles may be located remotely from the roles, assistant agents co-located with the roles have to be appointed to perform assisting activities. Accordingly, we distinguish between (functional) activities related to the selection of task resources (i.e. the selection of the vital signs (e.g. ECGs) and their quality) and the (physical) activities related to the acquisition of the required data (i.e. the selection, attachment and the calibration of the (physical) vital sign sensing devices, e.g. the selection of a high sensitive 3 leads ECG set in emergency services or the selection of chemical emission sensors in firefighting). In the healthcare context, the class media is task resource oriented and the class medium is device oriented (Figure 4).

At task resource level, the association class media-BAN-attachments models the assignments of a relevant and coherent set of medical data (i.e. aggregation of medium entities, incl. their quality) to the resource component of a task (Figure 4). This assignment has to be in line with the authorization rights of the role (dependence to class authorization represented by the dashed arrow in the figure). The class media-BAN-attachments also specify the relations between the media, for example, the priorities and the synchronization between the vital signs in emergency services or chemical emission measurement data in firefighting.

Analogously, device-BAN-attachments represent the correspondence of devices (e.g. vital sign sensors) to the (medical) data represented by the class medium. Especially relevant in healthcare, permissions to attach these devices also depend on the authorization rights of the role.

In a firefighting scenario, a two levels BAN assignments as proposed here can be used to equip a firefighter with emission sensors to enable an off-site member of the fire brigade to monitor fire exhaust for safety of the rescuers.

Both association classes are isomorphically connected to guarantee the correspondence between the devices and the vital signs (emission readings, resp.). Although a one-to-one association may model this isomorphism, we use two strongly connected homomorphisms to capture the dynamics of these attachments, the rippleup of device attachments to the task resource level (e.g. in case that the request for a device has been communicated out of band via an audio channel to the agent that attached the devices) and the ripple-down of medical media selections and attachments in case assignment has been initiated at task level (via a media-BAN attachments attribute), respectively.

The previously discussed separation of task-level and acquisition-level issues also provides a richer set of implementation options in a distributed environment of asymmetrical resource means with respect to wireless communication and computational processing resources. It enables the selection of (device/acquisitionlevel) communication alternatives available during disaster response that matches as 
closely as possible with the (task-level) facility specification, e.g. "use (and when needed hand-off to) an high fidelity voice conference facility" (grey colored construct in Figure 2 and cf. Hesselman (2003)). This separation is moreover common in healthcare standardization, which distinguishes between physical devices and virtual devices (Norgall, 2000).

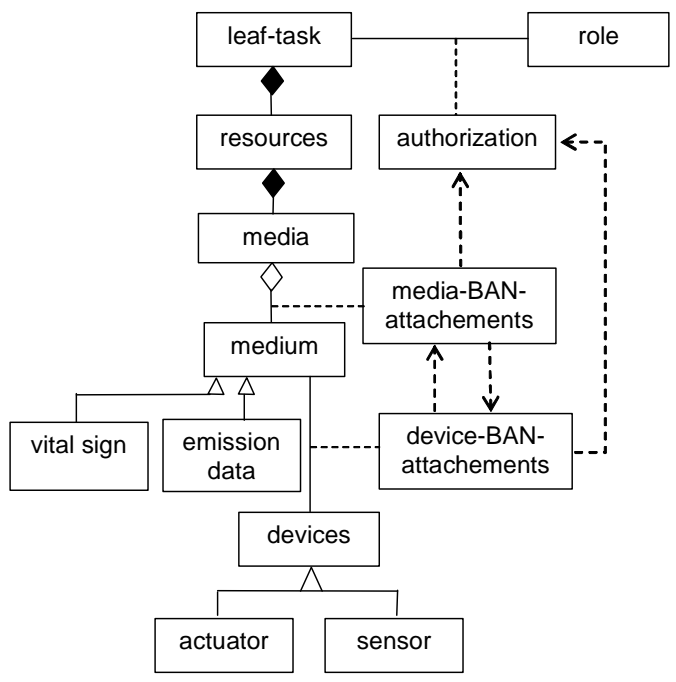

Figure 4 BAN based task resources and the assignment classes at task- and device-levels

\section{TELEMATIC NEEDS IN EMERGENCY AND RESCUE SERVICES}

To validate the benefits of the Enterprise models in the derivation of the requirements for the telematic system, we examine some of the issues in distributed management of disasters, in particular using the experience from the firework disaster. We also analyze the trauma team scenario valid in the Netherlands and used in the project MobiHealth (Jones et al., 2004). This project conducts healthcare trials to asses use of next generation wireless networks and mobile BANs for in- and outdoor healthcare.

\subsection{Trauma Team Scenario}

If a road accident happens, the Ambulance Coordination \& Dispatch Centre receives a request for ambulance assistance and contacts a regional ambulance service which serves that particular area. This service dispatches an ambulance to the 
scene of the accident with an "ambulance team" of paramedics on board. After a first assessment of the situation, the paramedics may request assistance from a mobile trauma team at a hospital and when considered necessary members of this team (e.g. an anaesthetist or a traumatologist) may travel to the scene in a second ambulance. In this scenario, we identify three sets of actors: the patient, the paramedics, and the members of the trauma team (at the HealthCare Centre and in the second ambulance).

Bi-directional audio-visual conversation facilities will be needed between the trauma team members and the paramedic or patient at the point of care. Moreover, upstream data transfer facilities will also be needed to convey vital signs of patients to the HealthCare Centre. On the other hand, low bandwidth downstream messaging services are required for command and control of the multimedia devices of paramedic headset, for example to move or zoom a paramedic's headset camera towards the patient or the scene of the accident to relieve the paramedics from burdening activities.

\subsection{Disaster Scenario}

The management of the firework disaster in Enschede in the Netherlands mentioned earlier did not only involve teams of different specialties (fire brigades, police, emergency services and coordination teams), it also operated within a layered organisational structure involving local, regional and national teams (Oosting, 2001). However, the regional and national teams had a facilitating role in the disaster response. In this response, the national police team deployed mobile communication units on the scene of the accident and local teams benefited from their experience with large-scale coordination and control.

The disaster initially started with harmless looking fires at the premise of the professional firework assembly site, but it evolved dramatically with two consecutive demolishing explosions. Before the explosions, management of the disaster operated adequately in terms of coordination and communication. The Alarm \& Dispatch Centre responded immediately by dispatching firefighting units, informed the Police Dispatch Room and the Ambulance Coordination \& Dispatch Centre. The on-site fire brigade officer and the ambulance paramedic correctly up-scaled the disaster management by requests for assistance, however, were unaware of the danger due to a lack of crucial premise-risk information.

Slow response of the Alarm \& Dispatch Centre to drastically up-scale the disaster response within half an hour after the explosions hampered efficient control, amongst others because afterwards the center became a bottleneck overloaded by public requests for help and also requests from off-duty rescue volunteers asking for information and instructions. Moreover, outcall traffic of the center was jammed amongst the burst public traffic in the public communication infrastructure. For 
example, resulting in a late on-site deployment of mobile communication units, while the national emergency network that interconnects important instances did not span over the site of the accident, which required a wireless infrastructure connected to the fixed emergency network.

In hospitals, first aid and medical trauma teams were quickly operational (e.g. 6 operating rooms were operational within half an hour). This due to the many off-duty staff that were alarmed by radio and television news bulletins, telephone calls from colleagues and also by the visibility of the disaster itself, the explosions and the large wreath of smoke. However, these teams passively wait for arriving patients and were unaware of what could be expected and how long to be on stand by after the burst of incoming patients. Even the hospital disaster management plan was instantiated on own initiative by the hospital without having received the request to do so by the Ambulance Coordination \& Dispatch Centre. Relevant feedback information for these medical teams came scarcely from news agencies, typically not under the scope of control of the crises coordination team until several hours after the explosion. The very seriously injured patients who arrived by personal cars or the patients who arrived by ambulance were treated adequately in accordance with the common working practices. However, the more serious cases amongst the many other patients who came individually using all available doors to enter chaotically the hospital were invisibly drowned within the large group of minimally traumatized patients.

Experience from management of this firework disaster reveals the following communication oriented needs:

- facilities for controlled deployment of off-duty rescue volunteers in a distributed environment;

- facilities for distributed inter and intra team coordination and communication, offand on-site, e.g. to acquire for an overview of the situation to improve efficient control and to enable up-scaling and also down-scaling of the disaster response;

- facilities to improve time-to-control of disaster situations in the envisioned augmented reality environment;

\section{TELEMATIC SUPPORT REQUIREMENTS}

This section describes the derivation of the requirements for the telematic system to support distributed disaster response. It mainly addresses the requirements that originate from the Enterprise models discussed earlier, in particular, the UML classes which associate entities that are possibly remotely located, e.g. teams and agents, devices and task resources. Healthcare processes in the setting of the trauma team scenario will be used as a vehicle to better illustrate the derivation and to extract concrete scenario specific requirements. Results are however valid for several other rescue processes as indicated in a later section. 
On the other hand, feasibility of the requirements depends on the capability constraints of the underlying ICT infrastructure, such as the constraints of a (public) wireless infrastructure and the limitation of BAN resources. This section therefore starts with a brief description of the infrastructure.

\subsection{Application Environment}

Figure 5 shows the Computational or Engineering level components of the healthcare system instantiated in a setting for the trauma team scenario. The MBUs (Mobile Base Units) are the gateways between patients' BAN vital sign sensing devices or the audio-video headset devices of the paramedic BAN and a secure networked infrastructure deployed on top of the public wired and wireless network infrastructure, such as the Internet and a providers' GPRS or UMTS infrastructure. The Surrogate Server (Dokovsky et al., 2003) intermediates as a high-level secure gateway between the HealthCare Centre node attached to a wired network and the BANs. Besides controlling secure interconnections this server among others bridges the performance hicks of the wireless communication services, as surrogate objects representing BANs run on this server.

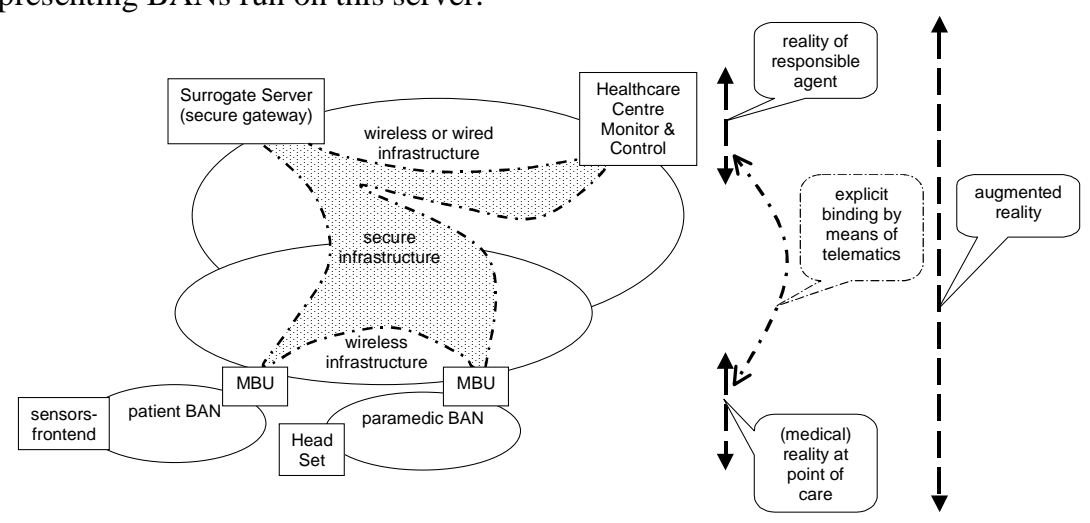

Figure 5 Mobile and wireless environment of the trauma case

In this setting, we observe the disjoint realities at the HealthCare Centre and at the point of care. Secure mobile and wireless telematic services bind the two realities onto an augmented reality, enabling availability of vital signs measured at the point of care at the HealthCare Centre.

The requirements for this distributed system derived from the (refined) Enterprise model, in particular the association classes media-BAN-attachments, device-BAN-attachments and (team to team) collaboration can be categorized amongst others into the following kinds: 
- Control-plane requirements: i.e. the requirements associated to the set-up of the bindings of the agent-to-agent communication and the remote monitoring of vital signs (or other types of monitored data in other rescue scenarios) that bridge the spatial gap between location of the role and the associated agent. This explicit binding involves the following issues:

o how to find the peer entity in the (wireless) distributed environment: e.g. naming \& addressing and discovery (or registration) issues;

o how to align the intentions: e.g. (negotiation) issues related to the readiness or willingness to set-up a binding;

o how to align the capability of the bindings: e.g. (negotiation) issues related to the type and the quality of service of the binding that matches to the requirements of the applications;

- Usage-plane requirements: i.e. the requirements associated to the use of the binding in respect of the coordination and communication activities in the application domain.

\subsection{Control-plane Requirements}

Some of the requirements for the BAN that are associated to the set-up of the augmented reality environment by an explicit binding of the remote point of care reality towards the scope of control of the off-site agent are the following:

Naming \& Addressing:

For a UML association class to specify which instantiations of class entities are associated, the instantiations have to be uniquely identifiable and addressable in the augmented reality environment. Accordingly, a device level BAN needs to be identifiable and addressable in the (wireless) network environment. Since a healthcare BAN discussed here is centrally controlled by an MBU, which also acts as a gateway, this MBU in turn needs to be addressable in the wireless environment. Furthermore, if the devices attached to the BAN need to be addressed individually and remotely, they have to have a unique address in the particular environment. For example, if a camera head-mounted on a paramedic can be addressed and controlled remotely, the paramedic will not be burdened by requests for adjustments of the camera settings. Moreover, each ECG channel of a multi-lead ECG setting has to be identified individually for correct interpretation by the responsible agents. Therefore, the data acquisition front-end of the devices needs to be addressed uniquely within a centrally controlled BAN, including each of the channels of this front-end.

Plug-and-Play Ripple-up:

If the MBU as a gateway is powered off, the BAN and its components are unknown in the networked environment. The MBU start-up should therefore contain 
a push mechanism (e.g. embodied by an announce or a registration protocol) to enable its discovery in the augmented reality environment.

Quality of Service mechanisms and multi-protocol systems may be further included to enable capability alignment within the network and the selection of available infrastructure technology (e.g. telephony, radiotelephony, GSM, UMTS) in case some of the systems ceased operation after a disaster.

Adaptable Communication:

In outdoor cases, communication resources are typically scarce. Amount, quality and coherence of the data to be exchanged have to match with the limitations of the communication channel capability. Set up of tailorable and adaptable telematic services is typically required in these environments to better cope with bandwidth limitations and variations in communication errors like data loss and channel dropouts. Buffers, data prioritising, synchronization mechanisms and data acknowledgements may be needed in these environments, including hand-off mechanisms to communication channels matching the task objective if alternative technologies are available at the scene (see also Figure 2).

\subsection{Usage-plane Requirements}

Although retrieval services in the form of a tightly coupled query-response mechanism satisfy remote monitoring applications, BAN intermittent availability and the way task resources are acquired (i.e. indirectly via collaboration of off-site and on-site agents), we prefer a less tightly coupled mechanism to retrieve BAN data.

Upstream Push Mechanism:

Dependence of media-BAN-attachments to device-BAN-attachments requires a push mechanism, such as a plug-and-play or a call-back mechanism, which enables the visualization (remote monitoring) of vital signs, for which a medical agent has attached corresponding sensors at a remote point of care. However, the agents may also communicate some vital sign data via an audio or video channel, e.g. patients' facial colour description as a trend vital sign for brain oxygenation (cyanosis).

Additionally, a (block-based) streaming protocol may be needed to upstream sets of coherent continuous-time vital signs or multimedia data. This also generates the need for several preservation mechanisms like (time and event) synchronization, data priority and data accuracy when using layered compression.

Downstream Messaging Mechanism:

Dependence of device-BAN-attachments to media-BAN-attachments also requires a messaging method that ripples down the choice for a specific task resource to the MBU to alert a medical agent or a patient at the remote point of care to 
attached a corresponding sensor or to forward a configuration setting to a sensor or actuator device. Remote camera control described earlier also emerges from this dependence.

\section{Conversational Communication:}

The requirements for the BAN in respect of the team collaboration or patient to team communication depend on the healthcare context. A GSM based channel often satisfies the requirements for communication between a paramedic and a trauma specialist in the trauma case. On the other hand, conversation between a patient and a specialist may need better fidelity. In chaotic or stressful situations, high fidelity audio channels help patients to calm down or be reassured, video channels may improve these conversations further.

\subsection{Refined Trauma Team Scenario Requirements}

The scenario described in the previous section also shows the interactivity of the communication between the remotely located agents including the transfer of the clinical patient data to the healthcare centre. This means that the data transfers are often dependent on one another and transfer delays therefore should not exceed the boundaries given by human factor studies. This constrains the audio-video communication quality in the wireless environment, it also constrains the quality of the vital signs which can be transferred. For example, an accurate 12 leads oversampled ECG set already requires a $64 \mathrm{kbits} / \mathrm{s}$ UMTS channel (approximately, 8 time-slots of GPRS Coding Schema 1) for real-time communication.

Moreover, several types of vital signs for emergency services are interdependent, together they form a unit of interpretation due to the typical indirect way of measuring patient condition. For example, oxygenation of the brain is usually estimated from oxygen saturation (O2sat) measured at a fingertip surface by beaming for blood colour, respiration rate including a $\mathrm{CO} 2$ (pCO2) measurement, temperature, blood pressure and heart rate or ECG measurements. However, as a first indication (trend sign) of oxygenation the patient's facial colour is observed for signs of cyanosis. In the estimation of brain oxygenation, validity of the measured O2sat depends on the patient's temperature. Moreover, heart rate as a trend sign of ECG has a higher priority than ECG. So the latter may be dropped or deferred in case of communication dips. The previously mentioned vital signs are typical for emergency services (Leisch and Orphanoudakis, 1999; Gagliano and Xiao, 1997).

Some other vital signs can be communicated by the paramedics to the trauma team at the HealthCare Centre via audio or video channels, for example pupil reaction, facial colour and also the (Glasgow Coma) trauma score. Other important information for a remotely located trauma team is still pictures or video of the scene of the accident to give an indication of patient's condition from the damage of the 
vehicle and the patient's positions in the vehicle. Today, polaroid pictures are taken and are handed over on the patient's arrival at the hospital A\&E department. Wireless transfer of these types of information from the scene will enable teams and facilities to be prepared before patient's arrival, and thus improve time to treatment of the patient on arrival at the hospital.

\subsection{Disaster Scenario Requirements}

Requirements for telematic support to facilitate the issues identified in the disaster scenario analysed are:

- Volunteering off-duty but on-site rescuers have to be identified immediately (i.e. assigned as agents) and deployed in teams to make best use of their availability. Uniquely addressable BANs that include telematic services for example with electronic registration services facilitate the deployment of these rescuers;

- Distributed intra and inter team coordination and communication is crucial in disaster control. Telematic services, mobile BANs of sensors and communicating devices and network infrastructure (incl. ad-hoc, wireless and possibly supported by mobile base stations) may facilitate this aspect. The telematic support requirements related to the two levels of BAN assignment discussed for the trauma team case is also suitable for distributed control in the case that an on-site rescuer carries devices controlled and monitored by off-site team members;

- Modelling separation of roles and agents enables on-site roles to be assigned to offsite agents. For example, an augmented reality environment enables the leader of the crises coordination team to visually receive an impression of the accident including statistic data while the person (e.g. the mayor of Enschede) is delayed in the traffic jam which often occurs in early stages of disasters.

- Dynamic improvement or fine-tuning of coordination and communication guidelines in up-scaling disasters may benefit from comprehensive enterprise models. Association classes and dependencies enable rippling through of new instructions towards roles or agents in the team hierarchy.

These telematic support requirements are similar to the requirements in the trauma team scenario case.

\section{CONCLUSIONS}

To achieve full control of disaster situations as rapidly as possible is a prime objective of disaster response. This chapter discusses the telematic service requirements for a mobile and wireless environment of BANs incorporating mobile devices (e.g. medical and multimedia devices) to support and to improve distributed disaster response in respect of time to control for example. These requirements are 
derived using a primarily top-down approach in-line with the ODP viewpoints framework.

The developed and proposed Enterprise models capture the organisational invariants of healthcare processes in UML compositional constructs in terms of roles, agents and tasks. This chapter emphasizes the modelling of roles and agents instead of tasks and task relations because neither process reengineering nor developing an information system for disaster management or healthcare is in the scope of the work. The modelling separation of roles and agents reveals the opportunities to apply telematic services because roles could be dispatched virtually to a point of care (or rescue) while some of the associated agents remained off-site at the command and control or healthcare center, yielding a simple form of an augmented reality environment. Further, the hierarchical structure of tasks and teams, flexibly constrained by association classes in the model, fits in scalable scenarios like disaster management and moreover, it retains, as much as possible, current ways of working of specialized teams.

The elaborated requirements of the trauma team scenario and the examined requirements for the more general disaster scenarios show the feasibility and added value of the mobile and wireless environment to improve rescue and emergency services. However, the derived requirements and the capabilities of the underlying mobile and wireless infrastructure seem to have mismatching characteristics. Provided (wireless) communication channels are often asymmetrical configured with a higher downstream bandwidth, but the addressed applications often require high bandwidth upstream channels. Moreover, the applications typically fetch data sets from the mobile devices, which have limited resources and are intermittently online. Internet protocols, which apply the client-server paradigm with typically a thin client and heavy server, could not be used in a straightforward manner in this inverted producer-consumer problem. A challenge for future work is amongst others the further development of a generic BAN which can be specialized for different specialties and the Internet protocol stack for the support of scalable multimedia services in networks comprising different technologies, including mechanisms to automatically discover alternative computing or network resources and select an alternative that matches with the task objective.

\section{REFERENCES}

Argyle, B., 1996, "New York Emergency Room RN: General Approach to Trauma", http://www.nyerrn.com/er/t/g.htm;

Auf der Heide, E., 1989, Disaster Response: Principles of Preparation and Coordination, CV Mosby St. Louis, on-line book at http://coe-dmha.org;

Azuma, R.T., 1997, “A Survey of Augmented Reality”, in Presence: Teleoperators and Virtual Environments, 6(4), pp. $355-385$; 
BBC News: world edition, 2002, "Failures in NY attack response", August $3^{\text {rd }}$, http://news.bbc.co.uk/2/hi/Americas/2170907.stm;

Blair, G., and Stefani, J-B., 1998, Open Distributed Processing and Multimedia, Addison-Wesley;

Booch, G., Rumbaugh, J., and Jacobson, I., 2001, The Unified Modeling Language User Guide, AddisonWesley;

Doarn, C.R., Nicogossian, A.E., and Merrell, R.C., "Telematic Support for Disaster Events", M-Health Emerging Mobile Health Systems, Istepanian, R.H., Laxminarayan, S., and Pattichis, C.S. (eds.), Kluwer Academic/Plenum Publ.;

Dokovsky, N., Halteren, van A., and Widya, I., 2003, "BANip: enabling remote healthcare monitoring with Body Area Networks", FIDJI2003 International Workshop on Scientific Engineering of Distributed Java Applications, Nov. 27-28, Luxembourg;

FEMA, 2001, FEMA Information Technology Architecture 2.0, http://www.fema.gov/library/ita.shtm;

Gagliano, D.M. and Xiao, Y., 1997, "Mobile Telemedicine Testbed" in Proc. 1997 American Medical Informatics association (AMIA) Annual Fall Symposium, pp.383- 387, National Library of Medicine Project N0-1-LM-6-3541;

Hesselman, C., Eertink, H., Widya, I. and Huizer, E., 2003, “A Mobility-aware Broadcasting Infrastructure for a Wireless Internet with Hotspots”, The $1^{\text {st }}$ ACM Int. Workshop on Wireless Mobile Applications and Services on WLAN Hotspots MWASH'03, P. Kermani (ed.), Sep. 19 ${ }^{\text {th }}$, San Diego CA, p.103-112;

Hobsley, M., 1979, Pathways in Surgical Management, Edward Arnolds Pub. Ltd, London;

Holz, E., Kath, O., and Born, M., 2001, "Manufacturing Software Components from Object-Oriented Design Models", $5^{\text {th }}$ IEEE Internat. Enterprise Distributed Object Computing Conference EDOC 2001, Sept $1^{\text {st }}$, Seatle, USA;

Jones, V. et al., 2004, "MobiHealth: Mobile Health Services based on Body Area Networks", M-Health Emerging Mobile Health Systems, Istepanian, R.H., Laxminarayan, S., and Pattichis, C.S. (eds.), Kluwer Academic/Plenum Publ.;

Leisch, E. and Orphanoudakis, S. 1999, "HECTOR Solutions" in 1st International Conference on Health Emergency Telematics, Sevilla, Spain, March 15-17, 17 pp.;

Luzi, D., Ricci, F.L., and Serbanati, L.D., 1997, "Extending the Standard Architecture for Healthcare Units: The Guideline Server", in New Technologies in Hospital Information Systems, J. Dudeck et al. (Eds.), IOS Press, pp. 95 - 101;

Meissner, A., Luckenbach, T., Risse, T., Kirste, T., and Kirchner, H., 2002, "Design Challenges for an Integrated Disaster Management Communication and Information System”, The 1st IEEE Workshop on Disaster Recovery Networks DIREN2002, June $24^{\text {th }}$, New York City NY;

Midkiff, S.F., and Bostian, C.W., 2002, "Rapidly-Deployable Broadband Wireless Networks for Disaster and Emergency Response", The 1st IEEE Workshop on Disaster Recovery Networks DIREN2002, June $24^{\text {th }}$, New York City NY;

Milgram, P. and Kishino, F., 1994, ”A Taxonomy of Mixed Reality Visual Displays”, IEICE Transactions on Information Systems, vol. E77-D, no. 12;

Norgall, T., 2000, "VITAL - towards a Family of Standards for Open Medical Device Communication" in XIII International Conference Computing in Clinical Laboratories, Milan, 21-23 September;

Oosting, 2001, "De Vuurwerkramp: Eindrapport", Commissie Onderzoek Vuurwerkramp, Enschede/den Haag, Feb. 28 ${ }^{\text {th }}$, ISBN: 90-71082-67-9, (in Dutch, incl. an English version of the summary "Final Consideration"), http://www.minbzk.nl/contents/pages/00001947/eindrapport_oosting_201.pdf;

Widya, I., Volman, C., Pokraev, S., De Diana, I. and Michiels, E., 2002, "Enterprise Modelling for an Educational Information Infrastructure", in Enterprise Information Systems III, J. Filipe et al. (eds.), Kluwer Academic Publ., pp. 231 - 239;

Yates, M., Takita, W., Demoudem, L., Jansson, and R., Mulder, H., 1997, “TINA Business Model and Reference Points - version 4.0", May 22 $2^{\text {nd }}$, http://www.tina.com; 